\title{
Communication Between Nurses and Physicians at Intensive Care Unit: A Comprehensive Review
}

\author{
Hanan Mohammed Mohammed :Assistant Professor of Medical-Surgical Nursing \\ Faculty of Nursing, Ain Shams University, Egypt
}

Dalia Abdallah: Assistant Professor of Medical-Surgical Nursing

Faculty of Nursing, Ain Shams University, Egypt

\begin{abstract}
:
Effective communication among healthcare professionals in the intensive care unit (ICU) is a particular imperative, with accurate and efficient interdisciplinary communication being a critical prerequisite for high-quality care. Nurses and physicians are highly important parts of the healthcare system workforce. Thus, identifying strategies that would improve communication between these two groups can provide evidence for practical improvement in the ICU, which will ultimately improve patient outcomes. This integrative literature review aimed to identify interventions that improve communication between nurses and physicians in ICUs. Although which intervention strategies are most effective remains unclear, this review suggests that these strategies improve communication to some extent. Future studies should be rigorously designed and outcome measures should be specific and validated to capture and reflect the effects of effective communication.
\end{abstract}

Keywords: Communication, Intervention, Intensive care units, Nurses and physicians 


\section{Tanta Scientific Nursing Journal}

\section{Introduction}

Improving quality and safety has become a priority for hospitals worldwide in recent decades. Effective communication among healthcare team members is one of the hallmarks of safe and highly reliable patient care [1]. Improving the communication between healthcare team members under rapidly changing social and medical conditions is becoming increasingly important.

Nurses and physicians are among the most important healthcare professional groups in hospital settings. They undertake separate and distinct tasks in clinical practice, yet they are expected to communicate effectively to provide effective services for patients [2]. Effective nurse-physician communication is a two-way process that involves sending the right message while being correctly received and understood by the other person ${ }^{[3]}$.

Effective nurse-physician communication has positive effects on the quality of patient outcomes, such as increased patient satisfaction $^{[4],}$ shortened length of stay (LOS) [5], and decreased adverse events [6]. On the contrary, ineffective nurse-physician communication may compromise patient safety and increase healthcare costs [7]. The
Joint Commission [1] reported that failure in communication causes two-thirds of sentinel events in healthcare. Dysfunctional communication accounts for $91 \%$ of the medical errors reported by resident physicians, which are linked with increased costs in healthcare institutions [6]. Poor nurse-physician communication may also lead to work dissatisfaction and lack of autonomy ${ }^{[8]}$ among nurses. Such working relationships have caused nurses to leave the profession $^{[8], ~ m a k i n g ~ r e t e n t i o n ~ a n d ~}$ recruitment of nurses increasingly difficult [5]. Physicians are reported to be easily frustrated when orders are not carried out timely. Unclear communication contributes to significant work dissatisfaction among physicians [1]. These influences on nurses and physicians all in turn affect the quality and safety of patient care.

In the modern healthcare system, the intensive care unit (ICU), which provides critically ill patients with high-quality care, is an essential component of largest hospitals. ICU patients are often critically ill and unstable, their clinical conditions change frequently, and their care often involves a multidisciplinary approach ${ }^{[4] . ~ I n ~}$ the complex environment of the ICU, the potential for adverse events is high ${ }^{[5] .}$ 


\section{Tanta Scientific Nursing Journal}

Effective communication between nurses and physicians is important for the safety and quality of patient care. Identifying strategies that would improve communication between these two groups could provide evidence for practical improvements in the ICU, which will ultimately improve patient outcomes. However, to our knowledge, no review conducted on interventions to improve communication between nurses and physicians in intensive care is available ${ }^{[8]}$.

\section{Barriers}

In addition to each profession's potential perceptions of the other, multiple barriers exist that hinder nurse-physician communication. A continuous flow of interruptions and multiple patient handoffs affect the ability of nurses and physicians to connect effectively, and establish a trusting and collegial relationship ${ }^{\text {[9]. }}$

Time is also a major factor in communication breakdown. Because nurses and physicians can be independently busy, finding time to communicate properly becomes a pressing. Work environments characterized by high patient acuity and staffing shortages create additional stress and thus contribute to communication breakdown. Even sex disparity among health care team members can create a barrier to effective communication ${ }^{[5]}$.

Males tend to prefer clear, quick, factbased communication, while females prefer a more in-depth discussion style that attempts to understand the reason for occurrences. Advances in technology implemented to increase quality and efficiency have a part in communication breakdown as well. Communication modalities, such as text pagers, patient inbox messaging, and electronic ordering systems, can contribute to increased errors. Use of these methods may misrepresent the urgency or the tone of the communication received; due to equipment malfunction, a message may not be received at all. Nurses and physicians express a desire to follow up on urgent orders or electronic messages with some form of verbal contact ${ }^{[10]}$.

\section{Historic Tension and Hierarchy}

The relationship between nurses and physicians has been characterized historically by hierarchy, power differential, and avoidance of open disagreement. Today, fundamental problems persist in many healthcare environments, including disruptive behavior by physicians, dismissive attitudes about nurses, and power and gender issues. Patient safety experts 


\section{Tanta Scientific Nursing Journal}

have pointed out the dangers that are associated with strict hierarchies in which individuals refrain from communicating concerns to those higher in the decisionmaking structure. As health reform pushes for greater accountability across the care continuum, healthcare providers will need to collaborate and function effectively on teams ${ }^{[11] .}$

\section{Divergent Views, Learned}

\section{Communication Style, and Terminology}

Professional education for nurses and physicians sets the stage for divergent views and perspectives. Nurses and physicians are trained to define well-being and its attainment differently. They are also taught to communicate very differently. Nurses are trained to relate information in narratives, whereas physicians are trained to provide the most concise, top-level communiqué possible. The fact that nurses and physicians are trained to communicate so differently can be a source of ongoing friction ${ }^{[6] \text {. }}$

In addition, nurses and physicians are trained under distinctly separate care models, which sometimes involve the use of different terminology to describe similar events. One of the key lessons learned by participants of the Idealized Design of Perinatal Care project convened by the
Institute for Healthcare Improvement was that physicians and nurses used different criteria, developed by their respective professional organizations, to assess and describe fetal monitoring patterns. An essential first step was developing a common language for describing the events[ ${ }^{12]}$.

\section{Solutions to Bring Nurses and}

\section{Physicians Together}

We believe that a three-pronged strategy is needed to improve ineffective communication between nurses and physicians: culture change, use of structured communication tools, and supportive technology. No one of these interventions, no matter how successfully applied, is sufficient. All three must be effectively implemented to optimize nurse-physician communication and avoid communication gaps that can lead to patient harm ${ }^{[11] .}$

\section{Culture Change}

The most fundamental intervention for improving nurse-physician communication is fostering an organizational culture that is patient-centric, safety-focused, and supportive of open communication and teamwork. Leaders play a crucial role in culture transformation by setting expectations, enabling and investing in 


\section{Tanta Scientific Nursing Journal}

specific structural supports, and modeling desired behaviors. As a means for improving nurse-physician communication, the Joint Commission recommends encouraging physicians to view patients as their primary customers and their role as partners in delivering the most effective and safe care. Focusing on the patient can bring purpose and meaning to the work of all clinicians and help reinforce the natural synergy

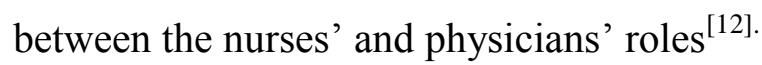
Leaders can support open communication and teamwork through several interventions. An essential first step is ensuring that adequate policies are in place for addressing disruptive physician behavior, a significant barrier to effective communication. Second is flattening the hierarchy within the organization and fostering respect among the various disciplines providing patient care. Regular teaching experiences provided by nurses for physicians and vice versa can help to personalize the nurse-physician relationship. In addition, specifically addressing the conflict between nurses and physicians can help prevent negative interpersonal dynamics ${ }^{[13] .}$

A third important intervention for open communication and teamwork is fostering the empowerment of nurses. By facilitating continuing education, participation on multidisciplinary committees, pursuit of specialty certification, and focused communication training, leaders can support nurses in communicating more confidently with physicians and other health professionals. Organizational leaders should consider pursuing Magnet designation as a means for improving the work environment for nurses. Nurses who work in hospitals that have achieved Magnet designation report higher quality relationships with physicians than peers who work in hospitals without Magnet status [14,15].

Finally, creating interdisciplinary patient care teams with a designated team manager sets the stage for teamwork and fosters improved communication. For example, an advanced practice nurse can serve as team manager; as such he or she is accountable for fostering timely communication between all care providers and the patient [6,9].

\section{Structured Communication Tools}

Specific communication tools have proven successful at improving communication among care providers. Developed by the Department of Defense and the Agency for Healthcare Research and Quality, Team STEPPS is a teamwork training program that focuses on the development of four core 


\section{Tanta Scientific Nursing Journal}

competencies: leadership, situation monitoring, mutual support, and communication. Participants are trained to use several team-based communication tools, including SBAR (see below), call outs (communicating verbally to other staff important decisions so they can anticipate next steps), and huddles (a brief face-to-face communication between care providers in which information is exchanged and the care plan is clarified). The process has been applied successfully in many healthcare settings, including the obstetrics unit [16].

SBAR (Situation, Background, Assessment, and Recommendation) is a structured communication tool that standardizes communication between health professionals. It can be especially effective when a nurse is contacting a physician with a concern about a change in patient status. By clearly spelling out his or her concerns, observations, interpretation, and recommendations, the nurse using SBAR provides the physician with a more complete picture of the clinical situation than might be the case without the tool. In this way, the use of SBAR can prevent the scenario in which the physician underestimates the significance of a clinical finding conveyed via telephone [10].
Similarly, tools such as a daily goals worksheet can be helpful in bridging the communication gap between busy nurses and physicians. Use of this tool in the ICU was associated with a significantly improved understanding of patient care goals among both nurses and physicians - and shorter ICU stays [17].

\section{Supportive Technology}

Technology solutions are essential for supporting effective communication between nurses and physicians. Two types of solutions are available: tools that enable a particular aspect of communication and software-based communication platforms that coordinate and standardize clinical communication. We believe both types of solutions are essential for effective nursephysician communication ${ }^{[18] .}$

To communicate effectively, clinicians must have reliable, secure communication tools. Email, text messages, and notes in the electronic medical record (EMR) facilitate asynchronous communication between nurses and physicians. An advantage of asynchronous communication, or communication between individuals who are not present at the same time, is that it may reduce interruptions, which have been shown to increase medical errors. To ensure 


\section{Tanta Scientific Nursing Journal}

compliance with Health Insurance Portability and Accountability Act (HIPAA) privacy regulations, these tools must be run on a secure network ${ }^{[19] .}$

Other tools facilitate direct, synchronous communication between nurses and physicians. A wireless, voice-controlled communication system enables nurses to contact physicians or other staff located within the hospital while remaining at the bedside. Worn as a badge pinned to the uniform or lab coat, the device can also send secure text messages and mobilize care teams. Use of handheld phones also allows nurses to remain at the bedside with the added benefit of being able to place calls to individuals outside the hospital ${ }^{[20] \text {. }}$

For truly effective, reliable communication between nurses and physicians, communication tools should be paired with a system-wide, software-based platform. A comprehensive communication platform standardizes the processes that underlie clinical communication and addresses the flow issues inherent in contacting physicians, whose practice workflow, call schedule, and contact preferences often change on a daily basis ${ }^{[21] .}$

A communication platform eliminates the need for nurses to remember physician- specific, frequently changing parameters every time they contact a physician. Instead, they call a single number and identify the physician they wish to contact by name or specialty. Using a rules-based algorithm, the platform initiates automated contact with the physician via the mode he or she has previously requested for that day and time. Return calls are automatically routed to the number designed by the caller ${ }^{[19]}$.

The combination of a communication platform that standardizes contact through reliable processes and secure, effective communication tools streamlines communication. For example, pairing a communication platform with handheld or smart phones allows nurses to quickly contact physicians - and await return callswithout leaving the bedside ${ }^{[21]}$.

\section{Conclusion}

The problem of ineffective nurse-physician communication is both common and complex. Multiple interrelated factors propagate the dynamic, which has clearly documented adverse effects on patient safety and other outcomes. Improving organizational culture, using structured communication tools, and linking a communication platform with secure, effective communication tools are critical 


\section{Tanta Scientific Nursing Journal}

for addressing the myriad communication gaps that thwart effective nurse-physician communication.

\section{References}

1. Weller, M. Boyd, D. Cumin Teams, tribes and patient safety: overcoming barriers to effective teamwork in healthcare Postgrad Med J 2014;1061(90); 149-154

2. K.J. O'Leary, N.L. Sehgal, G. Terrell, M.V. Williams Interdisciplinary teamwork in hospitals: a review and practical recommendations for improvement J Hosp Med2012; 7 (1): $48-54$

3. Courtney M. Effective Communication. Effective-Communication.Net. 2010. Available at: http://www.effectivecommunication.net/(Accessed on July 112014.

4. J.H. Larrabee, C.L. Ostrow, M.L. Withrow, M.A. Janney, G.R. Hobbs, C. Burant Predictors of patient satisfaction with inpatient hospital nursing care Res Nurs Health2016; 27 (4) :

5. D. Tschannen, B.J. Kalisch The impact of nurse/physician collaboration on patient length of stay J Nurs Manag, 2012;17 (7): 796-803
6. M. Riga, A. Vozikis, Y. Pollalis, K. Souliotis MERIS (Medical Error Reporting Information System) as an innovative patient safety intervention: a health policy perspective Health policy, $2015 ; 119$ (4). 539-548

7. T. Fassier, E. Azoulay Conflicts and communication gaps in the intensive care unit Curr Opin Crit Care 2010; 16 (6) :654-665

8. M. Williams, N. Hevelone, R.F. Alban, J.P. Hardy, D.A. Oxman, E. Garcia J, Measuring communication in the surgical ICU: better communication equals better care J Am Coll Surg, 2010;210 (1):. 17-22

9. A.F. Arriaga, A.W. Elbardissi, S.E. Regenbogen, C.C. Greenberg, W.R. Berry, S. Lipsitz, et al.A policy-based intervention for the reduction of communication breakdowns in inpatient surgical care: results from a Harvard surgical safety collaborative Ann Surg 2011; 253 (253). 849-854

10. J.M. Vardaman, P. Cornell, M.B. Gondo, J.M. Amis, M. TownsendGervis, C. Thetford Beyond communication: the role of standardized protocols in a changing 
health care environment Health Care

Manage Rev,2012; 37 (1): 88-97

11. The Joint Commission. Sentinel event data: root causes by event type. Retrieved from http://www.joint commission.org/assets/1/18/Root_Cau ses_Event_Type_2004-2Q_2015.pdf.

12. K.M. Sutcliffe, E. Lewton, M.M. Rosenthal Communication failures: an insidious contributor to medical mishaps Acad Med, 2017;79 (2):. 186-194

13. D.A. Saber Frontline registered nurse job satisfaction and predictors over three decades: a meta-analysis from 1980 to 2009 Nurs Outlook2014; 62 (6): 402-414

14. L. Zhang, L. Huang, M. Liu, H. Yan, X. Li Nurse-physician collaboration impacts job satisfaction and turnover among nurses: a hospital-based crosssectional study in Beijing Int $\mathbf{J}$ Nurs Pract, 2016;22 (3): 284-290

15. G.A. Nelson, M.L. King, S. Brodine Nurse-physician collaboration on medical-surgical units Medsurg Nurs,2017; 17 (1) : 35-40

16. A.H. Rosenstein Original research: nurse-physician relationships: impact on nurse satisfaction and retention Am J Nurs, 102 (6) (2002. 26-34
17. S. Thomson Nurse-physician collaboration: a comparison of the attitudes of nurses and physicians in the medical-surgical patient care setting Medsurg Nurse 2017; 16 (2): 87-91104

18. A.H. Rosenstein, M. O'Daniel Disruptive behavior and clinical outcomes: perceptions of nurses and physiciansAm J Nurs2011; 105 (1): 54-64

19. L.A. Despins Factors influencing when intensive care unit nurses go to the bedside to investigate patient related alarms: a descriptive qualitative study Intensive Crit Care Nurs, 2007;4 ( 3)

20. C.S. Hartog, J. Benbenishty Understanding nurse-physician conflicts in the ICU Intens Ccare Med, 2015;41 (2): 331-333

21. J.M. Rothschild, C.P. Landrigan, J.W. Cronin, R. Kaushal, S.W. Lockley, E. Burdick, et al. The Critical Care Safety Study: the incidence and nature of adverse events and serious medical errors in intensive care Crit Care Med, 2009;33 (8):. 1694-1700 